Archives de sciences sociales des religions

111 | juillet-septembre 2000

Varia

\title{
La mule et l'archevêque, ou la fortune des substituts
}

The mule and the Archbishop, or the fortune of substitutes

La mula y l'arzobispo, o la fortuna de los substitutos

Jean-Pierre Albert

\section{OpenEdition}

Journals

Édition électronique

URL : http://journals.openedition.org/assr/20229

DOI : $10.4000 /$ assr.20229

ISSN : $1777-5825$

Éditeur

Éditions de l'EHESS

Édition imprimée

Date de publication : 1 septembre 2000

Pagination : 221-234

ISBN : 2-222-96695-7

ISSN : 0335-5985

\section{Référence électronique}

Jean-Pierre Albert, "La mule et l'archevêque, ou la fortune des substituts », Archives de sciences

sociales des religions [En ligne], 111 | juillet-septembre 2000, mis en ligne le 30 mai 2016, consulté le 02 mai 2019. URL : http://journals.openedition.org/assr/20229 ; DOI : 10.4000/assr.20229

Ce document a été généré automatiquement le 2 mai 2019.

(c) Archives de sciences sociales des religions 


\title{
La mule et l'archevêque, ou la fortune des substituts
}

\author{
The mule and the Archbishop, or the fortune of substitutes \\ La mula y l'arzobispo, o la fortuna de los substitutos
}

Jean-Pierre Albert

1 Depuis que le groupe de recherche dont les contributions sont ici réunies a décidé de réfléchir sur la place des substituts dans le culte des saints, un passage de L'amour au temps du choléra de Gabriel Garcia Marquez me trotte dans la tête. Le voici (la scène se passe en Colombie, la région et l'hacienda dont il est question sont celles où vit l'héroïne du roman) :

«[...] l'archevêque de Riohacha se rendit dans la région en tournée pastorale, montant, sous un dais, sa célèbre mule blanche au tapis brodé d'or. Derrière lui marchaient des pèlerins venus de lointains villages, des joueurs d'accordéon, des vendeurs ambulants de victuailles et d'amulettes, et l'hacienda déborda trois jours durant d'invalides et de moribonds qui, en réalité, ne venaient pas pour les doctes sermons et les indulgences plénières de l'archevêque mais pour les faveurs de la mule dont on disait qu'elle accomplissait des miracles dans le dos de son maitre. »

Voilà donc une mule qui se substitue à un archevêque dans les attentes des fidèles et joue le même rôle qu'un saint vivant! J'ignore si l'anecdote est véridique, mais sa vérité anthropologique ne fait guère de doute à mes yeux. La mule et l'archevêque apparaissent en effet comme dispensateurs de biens de salut différents : du côté de l'institution, ils concernent l'autre monde - indulgences ou sermons porteurs de vérités salvatrices; du côté de la religiosité populaire, ce sont des miracles de guérison qui sont attendus. La mule ne prend pas seulement le pas sur l'archevêque en détournant vers elle l'attention, elle apporte quelque chose que l'institution en tant que telle ne peut offrir. Ce clivage recoupe assez bien celui qui traverse toute l'Église catholique entre, pour le dire en termes wébériens, un pôle sacerdotal (celui du rite, du charisme de fonction, de la médiation avec le Ciel déléguée aux seuls prêtres) et un pôle prophétique (celui du surgissement du surnaturel, du charisme personnel des saints). Et Garcia Marquez ne 
manque pas de noter la tension entre eux : la mule fait des miracles « dans le dos de son maître »...

Mais tension ne veut pas dire séparation. Si la mule peut être créditée de tant de pouvoir, cela tient pour partie à sa nature propre. Elle est plus proche du petit peuple, qui sait mieux comment se comporter avec une mule qu'avec un prélat; elle est blanche, chose plutôt rare et valorisable symboliquement. Encore faut-il qu'elle soit la mule de l'archevêque, et à ce titre harnachée d'un "tapis brodé d'or" qui contraste avec sa modeste condition de bête de somme ; à ce titre également impliquée dans des contextes rituels (la présence du dais, la procession) qui orientent immédiatement vers une lecture religieuse de la situation: je suppose que, quand elle est au pré, elle ne fait pas de miracles.

4 L'anecdote que l'on vient de lire - même si elle est imaginaire - est-elle représentative de certaines au moins des formules de substitution que l'on rencontre dans le catholicisme? Ici, un être impliqué dans un contexte rituel focalise les attentes des fidèles à la place de celui que l'on attendrait. C'est donc de façon un peu lâche que je rattache ce cas de figure à une problématique du substitut - on pourrait aussi légitimement parler de déplacement, au sens que Freud donne à ce terme dans son analyse du travail du rêve ou du symptôme : un objet devenant le support d'un investissement affectif qui concerne en réalité ce dont il occupe la place. Or il me semble que de tels déplacements des cibles d'investissements cultuels ne sont pas rares parmi les dévotions catholiques, en particulier dans ce qui touche au culte des saints. Sans doute s'agit-il de formes très particulières (et passablement hétérodoxes) de relations aux objets impliqués dans les cultes Mais il est vrai aussi que les liturgies les plus officielles font un grand usage d'objets - images, reliques, simulacres ${ }^{1}$ - que l'on peut sans peine faire figurer à la rubrique des « substituts » : une notion assurément très accueillante. J'essaierai pour commencer d'en préciser la signification ${ }^{2}$ en référence à des situations et des pratiques du culte catholique. Je tenterai ensuite de proposer une typologie sommaire des formes de substitution et de la mettre en relation avec quelques propriétés caractéristiques du champ religieux chrétien.

\section{Qu'est-ce qu'un substitut?}

5 Des choses - un signe, une image, un indice, un échantillon - ou des personnes - un représentant, un délégué, un porte-parole - peuvent être considérées comme des substituts. Dans tous ces cas, en effet, une réalité présente réfère à une réalité absente ou en quelque façon indisponible. Si une relation de ce genre n'est pas établie, l'objet donné à notre perception ne renvoie qu'à lui-même. Quelle peut être cette relation? Elle dépend, en premier lieu, d'un contexte d'usage. L'image d'un cheval vaudra comme substitut d'un cheval en chair et en os s'il s'agit de satisfaire une demande d'information du type : à quoi ressemble un cheval ? S'il s'agit de traîner une charrue, les substituts acceptables seront une mule ou un tracteur agricole. Il convient donc de définir ce que l'on attend dans un contexte de culte, afin de repérer à la fois le type de relation concerné (signification, équivalence fonctionnelle, représentativité, etc.) et le type de substitut adéquat.

6 En second lieu, parler d'un substitut donne à penser que l'objet ainsi désigné a une valeur moindre que celui dont il occupe la place, et qu'il est utilisé faute de mieux : à défaut de 
café, on recourt à de la chicorée ou à quelque autre succédané. Cette disqualification me semble caractéristique de la relation de substitution : il n'y aurait pas grand sens à dire, par exemple, que le mot vaut moins que la chose qu'il désigne. Certes, le cas des images est plus ambigu. La théologie chrétienne (catholique ou orthodoxe) a en effet intégré de longue date l'idée platonicienne d'une moindre valeur ontologique des images par rapport à leur référent sensible, ce dernier étant lui-même de moindre valeur que son prototype intelligible. Or si l'entité visée par le processus de substitution (au sens large) est de l'ordre du surnaturel, sa présentification à travers un objet de ce monde, et donc une réalité ontologiquement inférieure, est inévitable. L'attitude des fidèles par rapport à ce type d'objets de culte (reliques corporelles ou périphériques des saints, images, empreintes...) devrait donc être partagée entre la valorisation de l'élément matériel qui, en vérité, se donne dans l'expérience comme seul accessible et, d'un autre côté, la conscience de son imperfection. Cette contradiction se retrouve dans la théologie chrétienne de l'image ou, plus largement, des supports sensibles de la dévotion: en principe, ceux-ci sont simplement destinés à orienter la pensée vers le spirituel, ils ne sont que des intermédiaires permettant le dépassement de l'horizon de l'immanence, ce que la théologie chrétienne désigne parfois comme un transitus, un passage ou un franchissement. Mais les théologiens savent bien que ce processus peut échouer et se refermer sur une attitude « idolâtre ». En somme, il s'agit pour eux de fixer un bon usage du substitut : on concède à l'imparfaite nature de l'homme le recours à des intermédiaires sensibles dans ses relations avec le Ciel tout en mettant en garde contre une confusion entre l'objet de dévotion et son « prototype » surnaturel.

7 Adopter un point de vue normatif ne résout cependant pas ce qui reste une contradiction très problématique. D'un côté, en effet, l'objet-substitut doit évoquer avec vigueur son référent afin de susciter la vénération due à ce dernier et à lui seul; d'un autre côté, ce résultat ne peut être obtenu sans conférer au substitut lui-même des propriétés qui tendent à l'instituer en objet de culte légitime. L'Église catholique a voulu dépasser cette difficulté par des stratégies de compromis : d'accord pour les images, les reliques, mais sachons conserver la «bonne distance »- par exemple en rendant aux images un culte de dulie (révérence et dévotion) et non de latrie (adoration). Mais comment faire la différence dans les dévotions de masse ? Le terrain est glissant, et peu de chose sépare la dévotion légitime de la « superstition »...

Ce sont ces ambiguïtés qui nous occuperont ici, dans la mesure où, par-delà le relatif succès du contrôle institutionnel, le recours à des substituts dans le culte des saints ouvre large la porte à une créativité dévotionnelle considérable et souvent tenue pour suspecte. Certes, l'Église a tout à gagner à ce qu'un objet acquière le statut de "bon substitut ». Mais il n'y a sans doute pas d'accord entre le théologien rigoureux et le dévot ordinaire sur la définition de cette qualité. Je laisserai de côté pour le moment cette perspective normative afin de réfléchir en anthropologue (et non en théologien) sur ce qui qualifie un "bon substitut », c'est-à-dire un objet de dévotion efficace.

Il faut pour cela revenir à la question des contextes d'usage. Une pratique cultuelle vise, pour l'essentiel, à entrer en relation avec une entité surnaturelle afin d'en mobiliser la puissance. Dans le catholicisme, où la divinité et les autres entités impliquées dans un culte (anges, saints ${ }^{3}$, âmes des morts) sont de nature spirituelle - et donc en principe inaccessibles aux sens - la relation de communication établie avec le surnaturel passe le plus souvent, comme on vient de le voir, par un objet sensible qui sert de médiateur entre le dévot et son interlocuteur céleste. En fait, le culte officiel des saints n'implique a priori 
ni lieux ni objets obligés: une simple invocation par la prière suffit. Cette formule minimaliste se rencontre bel et bien dans la pratique ordinaire des croyants, quand ils invoquent les saints au fil des heurs et malheurs de la vie quotidienne. On la trouve également attestée dans les procès en canonisation lors de l'authentification des deux miracles nécessaires (toujours des guérisons miraculeuses) : étant donné qu'aucun culte autorisé n'existe encore, il est souhaitable que la demande d'intercession soit la plus économe possible en instrumentation cultuelle. Il reste que, dans ses formes dominantes, le culte des saints passe par des lieux, des reliques, des images. Il n'y a là rien d'hétérodoxe, sous la réserve mentionnée plus haut: que le support sensible de la dévotion dirige l'esprit du dévot vers son prototype intelligible. De fait, ce cheminement est assez spontané dans les cas de l'image et de la relique : hors de tout contexte religieux, la photographie d'une personne morte ou un objet lui ayant appartenu suffisent à évoquer sa mémoire. Or le culte des saints nous met souvent en présence de situations un peu différentes : il est des images et des reliques qui, dans certains contextes, semblent mieux que d'autres assurer la présence des saints en ce monde et capter leur puissance surnaturelle, au point de devenir des substituts actifs et presque autosuffisants. Ce résultat, comme on l'a dit, est à la fois voulu et non voulu par l'institution. Voyons à présent comment il devient possible.

\section{Quelques recettes pour construire de « bons » substituts}

10 Je présenterai pour commencer des formules qui, à défaut d'être délibérément adoptées, reçoivent l'aval de l'institution. Aval ne voulant pas dire contrôle : si l'Église peut faire des choix dans les objets-substituts qu'elle offre à la dévotion des fidèles, elle n'a qu'une prise limitée sur les modalités de leur réception.

\section{Évoquer un ailleurs}

11 La manière principale de construire un objet de culte, toujours présente en quelque façon dans les situations rituelles, consiste à attribuer à un objet des propriétés évoquant directement celles du surnaturel lui-même: c'est là le processus que l'on désigne en général à travers la notions de sacré ou de sacralisation. Les entités relevant d'un " autre monde » - dans notre culture : du surnaturel - sont toujours créditées de quelques traits définitionnels contre-intuitifs (ubiquité, omniscience, immortalité, etc.) qui sont autant de défis à nos usages ordinaires de la raison et nos savoirs empiriques les mieux assurés. Par exemple, les «mystères» du christianisme sont des énoncés explicitement revendiqués comme rétifs à toute élucidation rationnelle intégrale. Or il est tout à fait possible 1) de prêter à des objets sensibles des qualités de ce genre (en disant, par exemple, qu'une mince galette de pain azyme est de la chair humaine $\left.{ }^{4}\right)$; 2) d'agir en décalage avec les formes quotidiennes de l'action, dominées par la rationalité pragmatique : tel est justement l'espace du rituel. Ces deux stratégies sont en général associées. Ainsi, les justifications discursives sont relayées par les pratiques rituelles (on manipule l'hostie avec toute sorte de précautions et de gestes inutiles parce qu'elle est le corps du Christ) et, corollairement, la conscience de la ritualité favorise des hypothèses quant à la dimension surnaturelle des objets concernés (au minimum, l'idée que, si l'on agit comme on le fait, il doit y avoir une raison que la raison peine à connaître...). 

situations et des objets suscitant un trouble cognitif qui fait écho aux propriétés contreintuitives des êtres surnaturels ${ }^{5}$. S'agissant des objets, les anomalies définitionnelles peuvent êtres portées par le seul discours, comme dans le cas de la transsubstantiation. On les trouve aussi inscrites dans les choses elles-mêmes : par exemple, la plupart des reliques majeures de la croix sont retaillées ou montées en forme de croix, ce qui revient à superposer une relation de tout à partie à une relation analogique de représentation. Enfin, nombreux sont les cas de manifestation sensible d'un paradoxe ou d'une contradiction : le " presque rien » qu'est une hostie devient le centre des rituels fastueux de la Fête-Dieu, un ossement humain - matière normalement impure et répulsive - est constitué en objet de vénération... Autant de positions paradoxales de valeurs qui culminent, au grand scandale des anciens païens, dans le culte d'un crucifié.

\section{Signifier la médiation}

Second cas de figure: celui des moyens de signifier la médiation, le transitus, dans la construction sémantique et la mise en scène d'un objet offert au culte. On remarque, en premier lieu, que certaines reliques très valorisées - un bras du vieillard Siméon, conservé dans la basilique royale de Saint-Denis, l'index de la main droite de saint JeanBaptiste (dont il existe hélas plusieurs exemplaires), le noli me tangere, lambeau de chair momifiée qui subsiste sur le front du crâne supposé de Marie-Madeleine honoré à SaintMaximin, en Provence ${ }^{6}$ - sont des restes qui valent moins par leur lien métonymique avec le saint lui-même qu'en raison du contexte évangélique de leur qualification: dans les trois cas, ils évoquent un contact avec le Christ, ou du moins sa présence vivante. Siméon reçoit l'Enfant-Jésus dans ses bras lors de la Purification de Marie (Lc, 2, 25-28), le Baptiste désigne du doigt celui en qui il reconnaît le Sauveur lors du baptême dans le Jourdain (Jn, $\left.1,29^{7}\right)$, Marie-Madeleine est repoussée par Jésus à sa sortie du tombeau (Jn, 20, 17). Le sens de la relique se constitue donc dans un dépassement de sa pure perception comme objet (ce qui est le cas de figure habituel), mais aussi dans une réplication de la relation qui fonde sa valeur : de la relique au saint, du saint au Christ. N'y a-t-il pas là comme une pédagogie du transitus, une insistance sur la médiation en tant que telle qui convertit en sentiment d'une présence l'absence même du référent de la dévotion? Exhiber la relique, comme Suger le fit de celle de Siméon alors que la basilique de Saint-Denis, encore en chantier, était menacée par un orage épouvantable ${ }^{8}$, apparaît comme un moyen de convoquer le Christ en personne et de bénéficier directement de sa puissance salvatrice.

Un autre procédé rituel récurrent est la théâtralisation de la mise en présence du substitut, qui peut valoir comme « descente » ou apparition de l'entité surnaturelle ellemême. Marlène Albert-Llorca (2002) a développé l'analyse de ces rituels de dévoilement, de jeux sur l'absence et la présence dans la manipulation des images mariales. Les temps forts des liturgies autour des statues de la Vierge consistent, en règle générale, en la dramatisation de la disparition, puis de la « mise à disposition » d'une image par ailleurs accessible tout le reste de l'année dans son sanctuaire. On reconnait bien là la puissance du rite, déjà mentionnée comme toile de fond des situations qui nous intéressent : par son intermédiaire, c'est en somme l'accès à l'être surnaturel, ou sa venue parmi les hommes, qui se trouve assignée à un temps et un lieu, et constituée en un événement partagé par la foule des fidèles.

Archives de sciences sociales des religions, 111 | 2009 
15 La forme "événement », que l'on retrouve aussi dans les rites de passage biographique, semble donc apte à susciter l'intégration des significations religieuses au vécu des dévots. Elle cristallise dans une mise en présence construite par la dramaturgie du rite ce qui n'était au départ qu'un savoir abstrait (« il y a dans tel sanctuaire une relique de tel saint »). En somme, il faut " réveiller» les reliques, leur donner vie, les inscrire dans un processus d'entrée en contact qui suppose aussi une "événementialisation" de leur simple existence. La formule est certes d'une extrême généralité : tout culte organisé connaît des cérémonies, c'est-à-dire des temps, des lieux et des manipulations d'objets où s'actualise la représentation d'un contact avec le surnaturel. Or on constate que l'Église catholique a fait preuve d'une grande inventivité pour faire de l'événement avec des reliques : translations, processions, rites de baisement des reliquaires, etc. Dans tous les cas, il s'agit de dramatiser, à travers une mise en mouvement et/ou à disposition, un contact momentané, une présence exacerbée par les plages d'absence ou de distance qui l'entourent. Une relique ou une image sainte qui se contente d'être là tend à se fondre dans l'horizon unidimensionnel de l'objectalité. Pour ouvrir sur un ailleurs, il faut qu'elle bouge, qu'elle vienne et reparte, qu'elle vive. La preuve d'une porte, c'est qu'elle peut s'ouvrir. Une relique est une porte fermée que le rituel ou quelque autre mise en contexte doit ouvrir sur le Ciel.

Il faudrait mentionner ici tous les effets de cadrage qui s'ajoutent, de façon souvent redondante, à cette sémantique de la médiation avec l'au-delà, ou plus exactement en suscitent le mirage. Citons le choix des lieux de culte, souvent expressifs d'une logique de la frontière, du passage : soit qu'ils se trouvent sur des limites territoriales, soit dans des espaces évoquant une médiation entre le haut et le bas, etc. ${ }^{9}$ À noter également que les gestes de dévotion ajoutent encore à cette logique de la mise en présence : recherche d'un contact direct avec les réalités les plus suggestives, valorisation de l'itinéraire lui-même avec dramatisation de l'approche (ainsi les montjoies sur les itinéraires de pèlerinage), parcours processionnels ou programmes de dévotion mettant en scène une approche progressive du « saint des saints » (par exemple, lors des pèlerinages collectifs à Lourdes organisés par les diocèses ou d'autres institutions, c'est seulement le dernier jour de leur séjour que les pèlerins accèdent à la grotte).

17 Je n'irai pas plus loin dans cette énumération, dont on voit bien qu'elle nous met en présence de formules programmées par l'institution tout en ouvrant sur des pratiques beaucoup plus hétérodoxe : que penser, par exemple, des pèlerins qui déposent dans les anfractuosités de la grotte de Lourdes de petits papiers avec leur nom et leur demande? Des usages qui sont faits de l'eau de la source miraculeuse pieusement emportée chez soi dans un flacon en forme de Vierge, etc. ? Hors de tout point de vue normatif d'inspiration théologique, l'étude des substituts est une bonne ouverture vers une anthropologie des « superstitions ».

\section{Quand l'institution est débordée : le triomphe de la mule}

Les guillemets dont on encadre rituellement le mot superstition sont, ici comme bien souvent, l'indice d'une difficulté qui n'est pas que terminologique. Certes, le mot est moins utile que d'autres désignations vouées à la même prise de distance en sciences sociales des religions - le sacré, par exemple - et il est facile de lui substituer les termes plus neutres de dévotion, croyance ou pratique populaire (dans ce cas, c'est souvent le mot populaire qui hérite des guillemets). Il reste que, tout en faisant partie de ceux qui, 
depuis longtemps, ont contesté l'opposition tranchée entre orthodoxie et hétérodoxie, religion des élites et religion populaire, je suis sensible à la relative spécificité d'un style religieux qui n'est pas aussi soluble qu'on le voudrait dans le "théoriquement correct » des terminologies et les prudentes et évasives manifestations de distance : il y a là, à mon sens, quelque chose d'identifiable par delà les approximations ou orientations idéologiques du lexique.

Ce qui est en jeu, nous avons en fait différents moyens théoriques de le repérer : au minimum des hypothèses psychologiques inspirées de Freud (J.P. Albert, 1994) et une proposition sociologique adaptée de Max Weber. L'ordre de phénomènes visé par la catégorie de superstition a en effet beaucoup d'analogies avec ce que $M$. Weber désigne comme le domaine de la magie dans sa typologie des rôles et styles religieux. Il s'agit pour lui d'une strate, assez peu différenciée d'une religion à une autre, de demandes d'intervention du surnaturel dans le cours de la vie quotidienne qui passe par la médiation de spécialistes autres que les prêtres ou par la mise en œuvre de "recettes " connues de chacun. Dans tous les cas - et cela rejoint d'autres discours bien connus sur la magie - le rituel, la manipulation concrète d'objets et de substances joue un rôle central. La construction symbolique de l'efficacité de ces pratiques peut s'appuyer sur des ressources diverses, parmi lesquelles le recyclage de bribes de religions plus anciennes et le recours à des espaces culturels "autres" jouent un rôle non négligeable. Comme l'écrivent H. Hubert et M. Mauss (1950: 50), « la magie a parlé sanscrit dans l'Inde des pracrits, égyptien et hébreux dans le monde grec, grec dans le monde latin et latin chez nous ». Mais la religion légitime actuelle de la société considérée est une ressource tout aussi importante. Il semble que, dans l'Europe catholique post-tridentine ${ }^{10}$, ce dernier cas de figure soit au cœur des préoccupations de l'Église : ce n'est pas un hasard si le fameux traité de Jean-Baptiste Thiers s'intitule Traité des superstitions qui regardent les sacrements selon l'Écriture sainte. Il y est très largement question, en particulier, du mésusage des sacramentaux - de l'eau bénite au saint chrême - entrés de façon légitime ou illégitime en possession des fidèles.

Des pratiques de cet ordre existent donc dans des systèmes religieux dotés de spécialistes relevant de la catégorie wébérienne du sacerdoce. J'entends par là une formule dans laquelle une institution revendique avec un certain succès le monopole des relations avec le surnaturel et confie à ses propres spécialistes et à eux seuls l'organisation des cultes publics légitimes. Autrement dit, ce sont les prêtres, crédités d'un charisme de fonction, qui se trouvent en position de médiateurs obligés, ce qui revient à écarter (sauf cas particuliers) toute prétention individuelle à entrer directement en relations avec les entités surnaturelles et à en mobiliser la puissance. Or, face à une demande des fidèles qui fait une large place à des attentes plus terre à terre (guérison, chance, prospérité), ce type d'organisation a deux limites. D'une part, il est irréaliste : il y a toujours du religieux qui échappe à la sphère de la religion officielle - et ce d'autant plus lorsque, comme dans le catholicisme, on a affaire à une «religion de la transcendance » qui éloigne le Ciel de la terre et se veut centralement pourvoyeuse de biens de salut spirituels. D'autre part, il est à certains égards contre-productif pour l'institution elle-même, qui risque de s'enfermer dans un ritualisme peu attractif et a donc intérêt à signifier, en son sein même, la présence active du surnaturel.

21 Face à la première difficulté, le catholicisme a globalement manifesté un sens du compromis qui, outre qu'il a suscité bien des réactions internes, lui a souvent été reproché par un protestantisme plus rigoriste. On pourrait dire qu'il a lui-même organisé 
la diffusion d'objets (au XIX siècle : la Médaille miraculeuse, le bref de saint Antoine, et bien d'autres) dont on imagine mal des usages qui ne soient point «superstitieux » (y compris à ses propres yeux). Il s'agissait en somme de promouvoir une magie légitime, inscrite dans une pastorale en direction du «peuple» et jusqu'à un certain point contrôlable. Face à la seconde difficulté, l'élément le plus significatif est le culte des saints. Dans la typologie wébérienne, ceux-ci correspondent au pôle du prophétisme et permettent donc une mobilisation directe du surnaturel sans sortir du cadre religieux initial. A priori, les enjeux ne sont pas les mêmes que précédemment : il s'agit surtout de refonder dans l'actualité les capacités médiatrices d'une religion vouée, dans ses formes sacerdotales, à se priver des ressources de l'expérience (ou du spectacle) de la présence agissante du surnaturel.

Le pôle du prophétisme et celui de la magie (d'ailleurs clairement distingués par M. Weber) ne sont donc pas superposables : le premier est anti-ritualiste et vecteur de rationalité ${ }^{11}$, le second au contraire hyper-ritualiste et inscrit dans un monde enchanté. Mais ils ont en commun plusieurs traits structurels: la distance par rapport à l'institution, la capacité d'une mobilisation efficace du surnaturel. Ce rapprochement est encore plus sensible lorsqu'on passe de la vénération des saints vivants au culte des saints morts, grand pourvoyeur, on l'a dit, de pratiques magiques ou superstitieuses : ce dernier en effet entraîne la prolifération des substituts et par suite une focalisation sur des objets manipulables. Mais il concerne aussi les saints vivants, souvent promoteurs de nouveaux cultes, formules d'intercession, images inspirées par leurs visions, qui rappellent beaucoup les matériaux de la superstition et sont associés à des demandes (guérison, divination, liens avec les morts) clairement inscrites dans le registre de la magie au sens wébérien. Ajoutons enfin que le culte des saints, vivants ou morts, nourrit de plusieurs manières une marginalité, voire une ambivalence qui n'échappent pas à la conscience des acteurs. On a souvent souligné que des saints parfaitement reconnus par l'Église sont crédités par leurs dévots d'une « légende noire » qui revient à fonder leur puissance sur les forces du mal autant que celles du bien et les rend disponibles pour des manipulations que la morale réprouve : sainte Rita, saint Jude, saint Expédit à l'île de la Réunion ${ }^{12}$, etc. $\mathrm{Du}$ côté des saints vivants (ou de manifestations du surnaturel comme certaines apparitions mariales), l'absence initiale de reconnaissance par l'Église, voire même une condamnation explicite, ne sont pas un obstacle dirimant à la diffusion des cultes : elles en renforcent plutôt la marginalité et peuvent en cela les rendre encore plus attractifs.

Cet ensemble de données explique, me semble-t-il, les effets de déplacement (ou de focalisation "à côté ») dont la mule de G. Garcia Marquez nous a fourni le paradigme. Je n'en développerai ici qu'un seul exemple, celui du culte de saint Martin de Porres (1569-1639). Il s'agit typiquement d'un " petit saint », fils illégitime d'un noble espagnol et d'une esclave noire affranchie, dont la vie est toute marquée par une humilité hyperbolique: dans le couvent de Lima où il entra d'abord comme simple tertiaire dominicain, il était chargé de la cuisine, de la lessive, du ménage, il mettait ses talents de barbier au service de la communauté comme à celui des animaux pour lesquels il manifestait une infinie compassion... Ajoutons à cela des miracles insignes (bilocations, résurrections de morts) ou plus modestes, comme celui de débarrasser le couvent des souris qui rongeaient vêtements liturgiques et provisions de bouche en leur intimant l'ordre de rester dans le jardin, où il se chargeait de les nourrir. Ce type de profil, quoique rarement exploité dans le cadre du catholicisme, n'est pas dépourvu de vertus selon la logique chrétienne du renversement des valeurs du monde - éloge des humbles, pauvres 
de Dieu ou simples en esprit ${ }^{13}$. Mais, dans l'Espagne des années 1980 (je n'ai pas de données plus récentes), la dévotion populaire s'était focalisée sur un Martin expert en dératisation, dont le culte se polarisait sur les balais miniatures diffusés lors de sa fête. Autre trait significatif: saint Martin de Porres était invoqué à travers une chaîne de prières à recopier, "La peseta de la suerte de san Martin de Porres ", comportant une pièce d'une peseta collée sur chaque exemplaire avec du ruban adhésif et destinée à procurer bonheur et fortune ${ }^{14}$. Des pratiques semblables (qu'il s'agisse de chaînes diffusées par courrier ou déposées dans les églises) concernent, outre saint Antoine de Padoue, tous les saints " ambigus » évoqués plus haut. Il y a là, me semble-t-il, un triple déplacement: du saint selon l'Église à un personnage plus équivoque; du personnage à un objet qui cristallise son pouvoir; de la forme orale de la prière, manière normale d'invoquer les habitants de l'au-delà dans le catholicisme, à une forme écrite, en continuité avec les talismans, brefs et autres grimoires caractéristiques de la magie. En somme, cette logique de substitution a pour effet d'engendrer des objets " actifs » (pour ne pas dire : magiques) qui, tout en restant associés aux foyers de sacralité procurés par l'Église, parviennent à capter des forces plus obscures et à les rendre manipulables.

On peut se demander si ce processus de transgression plus ou moins consciente n'est pas à l'œuvre chaque fois qu'un objet de culte légitime est détourné de ses fonctions ou se trouve au centre d'un surinvestissement dévotionnel illégitime. Ce qui placerait la catégorie de substitut à la rencontre de deux mouvements de sens contraire : user d'un objet accessible à la place d'un autre, indisponible, mais aussi user autrement d'un objet disponible. Ce point d'arrivée n'a rien de surprenant si l'on se souvient que, dans le catholicisme, la question est moins celle du recours à des substituts - nécessaire et inévitable - que celle du rapport que l'on entretient avec eux. Les superstitions, d'ailleurs définies par la plupart des théologiens comme "vaines croyances" ou "vaines pratiques", sont en ce sens de "mauvaises manières" de se situer par rapport aux sacramentaux ou aux pratiques dévotionnelles. Leur identification implique donc un point de vue normatif : c'est moins le contenu des actes ou des croyances qui est en jeu que leur caractère licite ou illicite. Mais on peut se demander si cette distinction, au lieu d'être le simple effet d'un jugement extérieur de l'institution, n'est pas déjà active dans la motivation des gestes des croyants. E^tre superstitieux serait ainsi une tentative pour capter à son profit les puissances de l'au-delà, y compris celles relevant du «sacré gauche», qui viendrait s'ajouter, voire se substituer, aux formes autorisées de la dévotion.

\section{Pour conclure : une politique des substituts?}

J'ai examiné dans cette brève étude deux types de situation assez différents dans la manipulation d'objets dévotionnels: d'un côté le recours à des substituts proposés/ validés/tolérés par l'institution, de l'autre des formules rejetées comme idolâtres ou superstitieuses. Il s'agit là d'une polarité plus que d'une distinction impliquant des frontières tranchées, la rigueur des critères mis en œuvre ayant beaucoup varié dans le temps et dans l'espace. Depuis la crise iconoclaste du viII ${ }^{\mathrm{e}}$ siècle byzantin, le christianisme a connu périodiquement de semblables réactions rigoristes, à travers les Réformes $\mathrm{du} \mathrm{XvI}^{\mathrm{e}}$ siècle ou, plus près de nous, la mise en œuvre des réformes du second concile du Vatican. La réitération de ces gestes, que l'on pourrait aussi bien saisir au cas par cas, ou presque, à travers les rapports de visites pastorales établis par les évêques, me semble en vérité 
traduire un trait structurel de la vie d'une institution de type "Église »: la nécessité d'intégrer en quelque façon les ressources de la magie et du prophétisme. Dans les deux cas, les effets voulus de ces stratégies se mêlent de façon inextricable aux effets non voulus, voire aux effets pervers. Dans les deux cas, également, les problèmes se cristallisent autour de manipulations d'objets ou de rapports à des objets entrant pour la plupart dans la catégorie des substituts, images ou reliques au sens large ayant dans l'esprit des fidèles une relation de ressemblance, de contiguïté ou de causalité (pour parler comme Hume) avec les destinataires légitimes du culte. Les substituts concernés par une valorisation religieuse s'inscrivent en fait dans un continuum de décalages qui, d'un point de vue anthropologique, ne laisse apparaitre aucune différence qualitative entre ce qui sera déclaré licite ou illicite, dévotieux ou superstitieux, dogmatiquement correct ou hérétique. Et pourtant, ces catégorisations sont sans cesse réaffirmées parce qu'en elles se joue l'autorité de l'Église et sa prétention, toujours à renégocier, au monopole des transactions avec le surnaturel. Il s'agit là d'une question politique autant que religieuse, et cela explique à la fois le relatif succès et l'inévitable échec des interventions normatives de l'institution : incapable par principe de répondre à toutes les demandes, l'Église parvient néanmoins jusqu'à un certain point à les orienter vers des formes de recours à ses yeux légitimes. Mais, ce faisant, elle institue une frontière dont la transgression n'est pas sans attrait.

\section{BIBLIOGRAPHIE}

ALBERT Jean-Pierre, 1990, Odeurs de sainteté. La mythologie chrétienne des aromates, Paris, Éditions de l'École des hautes études en sciences sociales (réed. 1996 et 2004).

-, 1994 « La chaîne et la chance ", Archives de sciences sociales des religions, nº 86, p. 235-262.

,- 1997, Le sang et le Ciel. Les saintes mystiques dans le monde chrétien, Paris, Aubier.

-, 2000, « Des lieux où souffle l'Esprit », Archives de sciences sociales des religions, « Des formes sensibles de la religion ", $\mathrm{n}^{\circ} 111, \mathrm{p} .111-123$.

-, 2005, « Qui croit à la transsubstantiation? ", L'Homme, n 175-176, p. 369-396.

ALBERT-LlORCA Marlène, 2002, Les Vierges miraculeuses. Légendes et rituels, Paris, Gallimard.

BOYER Pascal, 1997, La religion comme phénomène naturel, Paris, Bayard.

-, 2001, Et l'homme créa les dieux. Comment expliquer la religion, Paris, Robert Laffont.

GARCIA MARQUEZ Gabriel, L'amour au temps du choléra, Paris, Grasset, 1987 [1985], p. 259.

Hubert Henri, Mauss Marcel, « Essai sur la magie », in Mauss M., Sociologie et anthropologie, Paris, Presses universitaires de France, 1950.

Mouton Marie-Dominique, 1997, « Le corps et ses substituts », Ateliers nº 18, p. 11-19.

SUGER, 1945, Comment fut construit Saint-Denis, éd. et trad. de J. Leclercq, Paris, Cerf.

WEBER Max, 1995 [1922], Économie et société, Paris, Plon, 2 vol. 


\section{NOTES}

1. Par exemple : un cénotaphe figurant le tombeau du Christ dans la liturgie pascale, un chemin de croix, une reproduction de la grotte de Lourdes...

2. Pour une mise au point sur la notion, voir M.-D. Mouton, 1997.

3. Je ne prends en compte ici que le culte officiel des saints, qui s'adresse toujours à des morts. Le cas des «saints vivants " pose des problèmes un peu différents dans la mesure où la vénération dont ils sont l'objet peut se passer de substituts.

4. Sur les facteurs subjectifs et le « cadrage » institutionnel de la crédibilité de cet énoncé, voir J.-

P. Albert, 2005.

5. Cette proposition théorique s'appuie essentiellement sur les ouvrages de Pascal Boyer (1997 et 2001).

6. Pour une courte bibliographie relative à ces cultes, voir J.-P. Albert, 1990.

7. En fait, dans cette scène comme dans la suivante, seules les paroles de Jean et de Jésus sont mentionnées. C'est la tradition (iconographique, en particulier) qui invoque un geste de désignation ou un contact.

8. Voir Suger, 1945.

9. Pour une synthèse critique sur l'anthropologie des lieux de culte, voir J.-P. Albert, 2000.

10. L'inquiétude face aux « survivances » des religions antérieures, même si elle n'est pas absente des sources européennes, persiste plus durablement dans les espaces coloniaux, en particulier en Amérique.

11. Cet aspect n'est pas toujours évident dans le message des saints chrétiens, à la différence du prophétisme juif. Il intervient cependant de façon certes paradoxale à travers la mystique, en ce qu'elle implique une valorisation de l'intériorité, soit une forme individualisée, intime et personnelle, de rapport au surnaturel compatible avec la logique religieuse de la modernité.

12. Le cas de sainte Rita est longuement analysé dans J.-P. Albert, 1997. Saint Jude est paradoxal en ce que son nom évoque Judas (leur nom est le même en Espagnol) et que certaines traditions le présentent comme un sosie du Christ. Sur le culte de saint Expédit à La Réunion, très impliqué dans des pratiques sorcellaires, voir le catalogue de l'exposition P'ti Bon Dieu, Musée agricole et industriel Stella Matutina, Piton-St-Leu, 1997.

13. Saint Martin de Porres, béatifié en 1836, n'a été canonisé qu'en 1962 par Jean XXIII. Il y avait dans ce geste le souci de doter les Amériques d'un nouveau saint (les saints américains sont encore très peu nombreux aujourd'hui) et aussi d'honorer un représentant des métis, dont saint Martin est devenu le patron.

14. Ce texte est analysé dans J.-P. Albert, 1994, et reproduit en annexe de l'article.

\section{RÉSUMÉS}

Cet article interroge le statut et le degré de légitimité des substituts dans l'Église catholique. Les substituts, qui apparaissent pour la plupart dans le culte des saints (il s'agit surtout d'images et de reliques), n'évoquent pas tous avec la même force l'entité qu'ils représentent. On a cherché à identifier quelques formules particulièrement efficaces à cet égard : référence à une situation de médiation, mise en présence ritualisée, construction d'événements dans lesquels la relique ou 
l'image est en position d'agent. Or ces stratégies, qui visent à renforcer le lien entre substitut et entité absente, ont pour effet paradoxal de mettre en valeur l'objet de culte dans sa matérialité. En conséquence, le bon usage des substituts s'oppose à leur autonomisation "superstitieuse », ce qui advient lorsqu'on les considère seulement en eux-mêmes, ou lorsqu'on cherche à capter la puissance que leur procure le caractère transgressif de leur culte.

This article examines the status and degree of legitimacy of substitutes in the Catholic Church. Substitutes, which appear mostly in the cult of saints (mostly images and relics), do not all mention with the same force the entity they represent. We sought to identify some particularly effective formulas in this regard: references to a situation of mediation, ritualized presence, construction of events in which a relic or image is in the position of agent. However, these strategies, which aim to strengthen the link between a substitute and an absent entity, have the paradoxical effect of enhancing the object of worship in its materiality. As a result, the proper use of substitutes opposes their "superstitious" empowerment, which happens when we consider them only in themselves, or when trying to capture the power that gives them the transgressive nature of their worship.

Este artículo se pregunta sobre el estatus y el grado de legitimidad que la Iglesia católica otorga a los substitutos. A menudo utilizados en el culto de los santos (son casi siempre imágenes y reliquias), no evocan siempre con la misma fuerza la entidad que representan. Se ha intentado identificar algunas fórmulas particularmente eficientes en esa perspectiva: referencia a una situación de mediación, escenificación ritualizada de la entidad, construcción de eventos en los que la imagen o la reliquia aparecen como agentes. Ahora bien, aunque la finalidad de esas estrategias sea reforzar el vínculo entre el substituto y la entidad ausente, tienen paradojalmente por resultado el de poner en valor la materialidad del objeto de culto. El uso correcto de los substitutos se opone pues a su autonomización "supersticiosa", lo que ocurre cuando son considerados solamente en sí mismos o cuando se intenta captar el poder que les da el carácter transgresivo de su culto.

\section{INDEX}

Mots-clés : image, relique, médiation, objet de culte, rituel, superstition

Palabras claves : imagen, reliquia, mediación, objeto de culto, superstición

Keywords : relic mediation object of worship, ritual

\section{AUTEUR}

\section{JEAN-PIERRE ALBERT}

Laboratoire interdisciplinaire solidarités sociétés territoires, Centre d'anthropologie sociale

(EHESS-CNRS, Université de Toulouse), albert@ehess.fr 